\title{
Bayesian analysis for lognormal distribution under progressive Type-II censoring
}

\author{
Sukhdev Singh ${ }^{1}$ (D), Yogesh Mani Tripathi ${ }^{1}$ (D), Shuo-Jye Wu² (D) \\ ${ }^{1}$ Department of Mathematics, Indian Institute of Technology Patna, Bihta - 801106, India \\ ${ }^{2}$ Department of Statistics, Tamkang University, Tamsui, New Taipei City 25137, Taiwan
}

\begin{abstract}
In this paper, we consider the problems of Bayesian estimation and prediction for lognormal distribution under progressive Type-II censored data. We propose various noninformative and informative priors for the unknown lognormal parameters and compute the Bayes estimates under squared error loss function. Importance sampling technique and OpenBUGS are taken into consideration for the computational purpose. Further, we predict lifetimes of both censored and future samples under one- and two-sample prediction frameworks. We also compute the corresponding Bayes predictive bounds. A simulation study is conducted to compare the performance of proposed estimates and a real data set is analyzed to illustrate applications of this study. Finally, a conclusion is presented.
\end{abstract}

Mathematics Subject Classification (2010). 62F15, 62N01, 62N05

Keywords. equal-tail interval, highest posterior density interval, one-sample prediction, OpenBUGS, two-sample prediction, importance sampling

\section{Introduction}

Censoring often occurs in many practical fields of studies such as life testing, reliability analysis, survival analysis, etc. Lawless [24] presented many useful applications of censoring in such studies and described that censoring can occur in a variety of ways. One may also refer to this text for a comprehensive review of the classical statistical methods applied to different censoring schemes. In this paper, we establish statistical inference for the lognormal distribution in presence of progressive Type-II censoring. This censoring has found extensive applications in life test studies and generalizes the traditional Type-II censoring. The interesting difference between these two censoring schemes is that under progressive Type-II censoring, live units can be removed during the experimentation as opposed to the Type-II censoring, where removal of units can occur only at the end of the experiment. Several researchers have analyzed many lifetime models under this censoring, and the recent treatise by Balakrishnan and Cramer [6] includes many important results in this aspect. In addition, one may also refer to $\mathrm{Ng}$ et al. [30], Kundu [21], Pradhan

\footnotetext{
*Corresponding Author.

Email addresses: sukhdev@iitp.ac.in (S. Singh), yogesh@iitp.ac.in (Y. M. Tripathi), shuo@stat.tku.edu.tw (S.-J. Wu)

Received: 12.04.2017; Accepted: 28.11.2018
} 
and Kundu [32], Huang and $\mathrm{Wu}$ [14], Dey et al. [13], and references cited there-in for a detailed review of work done on this particular censoring.

Lognormal distribution is used to model particle size distributions, biological specimens distribution, RNA-Sequencing, etc. It has also found wide applications in the studies of degradation phenomena for a number of electrical and mechanical processes. In fact, Leipnik [25] observed that various investigations in telecommunication and radar engineering lead to the study of properties of a lognormal distribution. One may also refer to Johnson et al. [18] for several applications of this distribution in various life testing experiments. This distribution is treated as an alternative model to some known distributions like Weibull, gamma and generalized exponential models, see for instance, Basavalingappa et al. [10], Raqab et al. [33], and Jia et al. [17]. Crow and Shimizu [12] discussed the importance of Bayesian inference for the lognormal distribution in various life testing studies.

In statistical analysis, one emphasizes generally on finding efficient classical and Bayesian estimates for the unknown parameters of an underlying population. However, an essential and important feature of various life test studies including clinical trials, quality control, agricultural and industrial experiments is to predict future observable under some partial information. Sometimes it is also of interest to provide one- and/or two-sided bounds on these unknown random quantities. Many examples abound in practice and we refer to Meeker and Escobar [27] for some useful implications of such studies. We further notice that a very rich literature exists on predictive inference under both classical and Bayesian approaches. In the present work, we mainly focus on finding predictive estimates from the Bayesian perspective. Bayesian prediction and associated inference for future lifetimes based on the censored data have been widely studied by many authors. Al-Hussaini [1] obtained predictive estimates for a general class of distributions which include Weibull, Pareto, beta, Gompertz and compound Gompertz distributions under Bayesian framework when it is known that samples are Type-II censored. One may also refer to Kundu and Howlader [22], Kundu and Raqab [23], and Panahi and Sayyareh [31] for some more interesting results in this aspect. Several researchers have also discussed the problem of Bayesian prediction under progressive Type-II censoring, and one may refer to Ali Mousa [2] for Pareto distribution, Ali Mousa and Jaheen [4] for Burr distribution, Jaheen [16] for Gompertz distribution, Soliman [38] for Burr Type-XII distribution, Ali Mousa and Al-Sagheer [3] for Rayleigh distribution, and Huang and Wu [14] for Weibull distribution. One may also refer to Mohie El-Din and Shafay [28] for some more interesting results in this regard.

Some related classical inferences upon unknown parameters of a normal distribution under progressive Type-II censoring are derived by Balakrishnan et al. [7] and $\mathrm{Ng}$ et al. [29]. Particularly, authors have discussed maximum likelihood and approximate maximum likelihood estimates along with asymptotic intervals of normal parameters. Some related inference can also be found in the article by Balakrishnan and Mi [8]. Recently, Singh et al. [36] derived various point and intervals estimates for this distribution using progressive Type-II censored samples. Authors have adopted both the classical and Bayesian methods in this work.

The main purpose of the present work is to obtain Bayesian prediction and the corresponding prediction bounds for the censored and future observations of progressive Type-II data. The rest of this paper is organized as follows. We discuss progressive Type-II censoring and some basic properties of lognormal distribution in Section 2. Various posterior densities are obtained in Section 3. Bayesian analysis is presented in Section 4. Section 5 deals with the Bayesian prediction. A real data analysis and a simulation study are conducted in Section 6. Finally, we present some concluding remarks in Section 7. 


\section{Preliminaries}

In this section, we briefly describe progressive Type-II censoring and also discuss some basic properties of the lognormal distribution. Let lifetimes of some experimental units put on a test follow a continuous distribution with probability density function (PDF) and cumulative distribution function $(\mathrm{CDF})$ given by $f(\cdot ; \theta)$ and $F(\cdot ; \theta)$, respectively. Here $\theta$ is a vector of unknown parameters of the distribution. In progressive Type-II censoring, a total of $n$ test units is placed on a life test and the experiment is terminated after observing a prescribed number of failures, say $m$. Thus a progressive Type-II censored sample of size $m$ can be obtained in $m$ stages as described below. At the time of first failure, say, $x_{(1)}, R_{1}$ number of units are randomly removed from the remaining $n-1$ surviving units. Similarly, $R_{2}$ number of units are randomly removed from the remaining $n-R_{1}-2$ surviving units at the time of second failure $x_{(2)}$ and so on. Finally, when $m$-th failure at time $x_{(m)}$ is observed, all the remaining $R_{m}=n-\sum_{i=1}^{m-1} R_{i}-m$ units are removed from the experiment and the test is terminated. Thus, $\left(x_{(1)}, x_{(2)}, \ldots, x_{(m)}\right)$ represents a progressive Type-II censored sample of size $m$ corresponding to censoring scheme $\boldsymbol{R}=\left(R_{1}, R_{2}, \ldots, R_{m}\right)$. Here censoring scheme $\boldsymbol{R}$ is prefixed prior to the start of the experiment such that $\sum_{i=1}^{m} R_{i}=$ $n-m$. Observe that progressive Type-II censoring reduces to the traditional Type-II censoring when $R_{i}=0, i=1,2, \ldots, m-1$, and $R_{m}=n-m$. The likelihood function of $\theta$ given the progressive Type-II censored data $\boldsymbol{x}=\left(x_{(1)}, x_{(2)}, \ldots, x_{(m)}\right)$ can be written as

$$
l(\theta \mid \boldsymbol{x})=C \prod_{i=1}^{m} f\left(x_{(i)} ; \theta\right)\left[1-F\left(x_{(i)} ; \theta\right)\right]^{R_{i}},
$$

where $C=n\left(n-R_{1}-1\right)\left(n-R_{1}-R_{2}-2\right) \ldots\left(n-\sum_{i=1}^{m-1} R_{i}-m\right)$.

A random variable $X$ following lognormal $L N(\mu, \tau)$ distribution has PDF and CDF of the form

$$
f(x ; \mu, \tau)=\frac{1}{x \sqrt{\tau}} \phi\left(\frac{\ln x-\mu}{\sqrt{\tau}}\right), \quad x>0, \quad-\infty<\mu<\infty, \quad \tau>0,
$$

and

$$
F(x ; \mu, \tau)=\Phi\left(\frac{\ln x-\mu}{\sqrt{\tau}}\right),
$$

where $\phi(\cdot)$ and $\Phi(\cdot)$, respectively, denote the density and the distribution function of the standard normal random variable. Moreover, $\mu$ and $\tau$ are the parameters of this distribution. In fact, $\tau$ governs the shape of this distribution and furthermore, it is an uni-modal distribution with mode given by $e^{\mu-\tau}$. In general, the hazard function of a two-parameter lognormal distribution first increases up to some time point $x,(x>0)$ and then decreases to zero. We further notice that for large $\tau$, hazard function is practically a decreasing function in $x$. One may further refer to Crow and Shimizu [12] for several applications of this distribution in lifetime analysis.

\section{Posterior distributions}

Suppose that $\boldsymbol{x}=\left(x_{(1)}, x_{(2)}, \ldots, x_{(m)}\right)$ is a progressive Type-II censored sample of size $m$ taken from a distribution $f(x ; \theta)$. Now, assume that $\pi(\theta)$ denotes a prior of the unknown parameter $\theta$. Then, using the likelihood function $l(\theta \mid \boldsymbol{x})$, the corresponding posterior density can be obtained as

$$
\pi(\theta \mid \boldsymbol{x})=\frac{\pi(\theta) l(\theta \mid \boldsymbol{x})}{\int_{\Theta} \pi(\theta) l(\theta \mid \boldsymbol{x}) d \theta}, \quad \theta \in \Theta .
$$

In this section, we consider various informative and non-informative priors for the unknown parameters of a $L N(\mu, \tau)$ distribution. Several authors have analyzed many lifetime models assuming different priors for unknown quantities of interest and one may refer to Sinha 
[37] for a detailed discussion on these accounts. For our model, we obtain posterior densities for the cases when (i) $\mu$ is unknown, (ii) $\tau$ is unknown, and (iii) both $\mu$ and $\tau$ are unknown. For the sake of convenience, we denote the corresponding non-informative and informative priors by $\pi_{i}^{*}(\theta)$ and $\pi_{i}(\theta)$ respectively, $i=1,2,3$.

\section{1. $\mu$ is unknown}

In this case, we treat $L N(\mu, \tau)$ distribution as a one-parameter distribution. We assume that the parameter $\tau$ is known. We first take a non-informative prior for $\mu$ as $\pi_{1}^{*}(\mu)=1$, $-\infty<\mu<\infty$, which is uniform over the full parameter space. The corresponding posterior density is obtained as

$$
\pi_{1}^{*}(\mu \mid \boldsymbol{x}) \propto N_{\mu}\left(\frac{\sum_{i=1}^{m} \ln x_{(i)}}{m}, \frac{\tau}{m}\right) h(\mu, \tau)
$$

Here $h(\mu, \tau)=\prod_{i=1}^{m}\left[1-\Phi\left(\frac{\ln x_{(i)}-\mu}{\sqrt{\tau}}\right)\right]^{R_{i}}$, and the PDF of a normal distribution is represented by $N_{\mu}(\cdot, \cdot)$. Further, we consider an informative prior $\pi_{1}(\mu)=N_{\mu}\left(a_{1}, b_{1}\right)$ for $\mu$ in which $a_{1}$ and $b_{1}$ reflect the prior knowledge about $\mu$. Under this prior, the associated posterior density is given by

$$
\pi_{1}(\mu \mid \boldsymbol{x}) \propto N_{\mu}\left(\frac{a_{1} \tau+b_{1} \sum_{i=1}^{m} \ln x_{(i)}}{\tau+b_{1} m}, \frac{b_{1} \tau}{b_{1} m+\tau}\right) h(\mu, \tau) .
$$

\section{2. $\tau$ is unknown}

Here $\mu$ is assumed to be a known parameter and $\tau$ is treated as an unknown parameter. We propose an inverse gamma $I G\left(p_{2}, q_{2} / 2\right)$ distribution as a proper prior for $\tau$ and denote it by $\pi_{2}(\tau)$ where

$$
\pi_{2}\left(\tau ; p_{2}, q_{2}\right) \propto \tau^{-\left(p_{2}+1\right)} e^{-\frac{q_{2}}{\tau}}, \quad \tau>0, p_{2}>0, q_{2}>0 .
$$

The corresponding posterior density is given by

$$
\pi_{2}(\tau \mid \boldsymbol{x}) \propto I G_{\tau}\left(\frac{m}{2}+p_{2}, 0.5\left(q_{2}+\sum_{i=1}^{m}\left(\ln x_{(i)}-\mu\right)^{2}\right)\right) h(\mu, \tau) .
$$

We further notice that $\pi_{2}(\tau)$ is quite flexible class of priors. For instance, this class of priors includes a non-informative prior like $\pi_{2}^{*}(\tau)=\frac{1}{\tau}, \tau>0$ which corresponds to the case when hyper-parameters are assigned values as $p_{2}=q_{2}=0$. Such type of priors have been widely discussed in the literature and one may refer to Khan et al. [20] and Singh and Tripathi [35], and the references cited there-in for further details.

\section{3. $\mu$ and $\tau$ are unknown}

In this section, both the parameters $\mu$ and $\tau$ are assumed to be unknown. Several researchers have proposed different prior distributions for the parameters of various lifetime models of interest. For instance, Kundu [21] and Banerjee and Kundu [9] proposed gamma priors for unknown shape and scale parameters of a Weibull distribution, see also Asgharzadeh et al. [5]. Huang and $\mathrm{Wu}$ [14] considered a bivariate prior for unknown scale and shape parameters of the Weibull distribution. We have taken into account the non-informative Jeffreys prior for unknown parameters of the lognormal distribution. Similarly, a bivariate proper prior is taken in to consideration for deriving the desired inference. One may refer to Crow and Shimizu [12] and Singh and Tripathi [34] for a discussion on 
such type of prior distributions. Observe that with the Jeffreys prior, $\pi_{3}^{*}(\mu, \tau)=1 / \tau$, $-\infty<\mu<\infty, 0<\tau<\infty$ for $(\mu, \tau)$, the associated posterior density turns out to be

$$
\begin{gathered}
\pi_{3}^{*}(\mu, \tau \mid \boldsymbol{x}) \propto \quad I G_{\tau}\left(\frac{m-1}{2}, \frac{1}{2}\left(\sum_{i=1}^{m}\left(\ln x_{(i)}\right)^{2}-\frac{\left(\sum_{i=1}^{m} \ln x_{(i)}\right)^{2}}{m}\right)\right) \\
N_{\mu \mid \tau}\left(\frac{\sum_{i=1}^{m} \ln x_{(i)}}{m}, \frac{\tau}{m}\right) h(\mu, \tau) .
\end{gathered}
$$

Next, we consider a conditional bivariate prior for $(\mu, \tau)$ as

$$
\pi_{3}(\mu, \tau)=\pi(\tau) \pi(\mu \mid \tau),
$$

where $\pi(\tau)$ and $\pi(\mu \mid \tau)$, respectively, denote $I G\left(p_{2}, q_{2} / 2\right)$ and $N\left(a_{1}, \tau / b_{1}\right)$ distributions. The posterior density is

$$
\begin{gathered}
\pi_{3}(\mu, \tau \mid \boldsymbol{x}) \propto I G_{\tau}\left(\frac{m}{2}+p_{2}, \frac{1}{2}\left\{\sum_{i=1}^{m}\left(\ln x_{(i)}\right)^{2}+a_{1}^{2} b_{1}+q_{2}-\frac{\left(\sum_{i=1}^{m} \ln x_{(i)}+a_{1} b_{1}\right)^{2}}{m+b_{1}}\right\}\right) \\
N_{\mu \mid \tau}\left(\frac{\sum_{i=1}^{m} \ln x_{(i)}+a_{1} b_{1}}{m+b_{1}}, \frac{\tau}{m+b_{1}}\right) h(\mu, \tau) .
\end{gathered}
$$

\section{Bayesian estimation}

This section deals with Bayesian analysis of unknown parameters of the $L N(\mu, \tau)$ distribution. Notice that, in Bayesian analysis, the Bayes estimates of a parametric function $g(\theta)$ can be obtained under a prescribed loss function. In the present work, we consider the squared error loss, however, other loss function can also be incorporated. We mention that, under squared error loss, the Bayes estimate of $g(\theta)$ is the posterior mean and is obtained as

$$
\hat{g}_{B}(\theta)=\int_{\Theta} g(\theta) \pi(\theta \mid \boldsymbol{x}) d \theta .
$$

Now, it is seen that under the posterior densities discussed in previous section, the above expression can not be solved in a closed form. Therefore, some approximation techniques such as Lindley's method (see, Lindley [26]) and Tierney and Kadane method (see, Tierney and Kadane [39]) can be used to obtain the approximate Bayes estimates. However, using any of the two methods, the associated Bayesian credible intervals for the unknown lognormal parameters can not be obtained. Therefore, we propose importance sampling technique which is a useful method for generating random samples from various posterior densities, in order to compute the desired Bayes estimates. Observe that various posterior densities obtained in Section 3 appear to be in the form of

$$
\pi(\theta \mid \boldsymbol{x}) \propto f_{\theta}\left(c_{1}, c_{2}\right) h(\theta),
$$

where $f_{\theta}\left(c_{1}, c_{2}\right)$ represents some known distribution of $\theta$ with $c_{1}, c_{2}$ being the corresponding hyper-parameters. Therefore, using importance sampling technique and under squared error loss the desired Bayes estimate of $g(\theta)$ is obtained as

$$
\hat{g}_{B}=\frac{\sum_{i=1}^{s} g\left(\theta_{i}\right) h\left(\theta_{i}\right)}{\sum_{i=1}^{s} h\left(\theta_{i}\right)},
$$

where $\left\{\theta_{i}: i=1,2, \ldots, s\right\}$ denotes a random sample from the distribution $f_{\theta}\left(c_{1}, c_{2}\right)$. More precisely, let us consider the prior as given by Equation (3.1). Then, the samples from the posterior density given by Equation (3.2) can be generated as follows.

Step 1: Generate $\tau \sim I G_{\tau}(\cdot, \cdot)$ and $\mu \sim N_{\mu \mid \tau}(\cdot, \cdot) \quad$ (see, Equation (3.2)).

Step 2: Repeat Step 1, s times to generate samples like $\left(\mu_{1}, \tau_{1}\right),\left(\mu_{2}, \tau_{2}\right), \ldots$, $\left(\mu_{s}, \tau_{s}\right)$. 
Further, the generated samples together with the method of Chen and Shao [11] can be taken into consideration to obtain the corresponding Bayesian credible interval. One may also refer to Singh et al. [36] for the details of this method. It is to be mentioned that, in the present work, the corresponding posterior densities turn out to be in tractable form so we were able to apply the importance sampling technique. But, under any other prior, it may or may not be possible, for an example if one considers independent priors for $\mu$ and $\tau$ such as $\pi(\mu)=N\left(a_{1}, b_{1}\right)$ and $\pi(\tau)=I G\left(p_{2}, q_{2}\right)$. In that case, the posterior density does not turn out to be in tractable form so the proposed importance sampling technique can not be used. Hence, the Bayes estimates and corresponding Bayesian credible intervals can not be obtained using the importance sampling. Therefore, under such class of priors and also for other proposed priors, we further consider OpenBUGS software for Bayesian analysis. The BUGS (Bayesian inference Using Gibbs Sampling) software determines an appropriate MCMC scheme (based on the Gibbs sampler) for analyzing the specified model. The Bayes estimates and corresponding Bayesian credible interval estimates can be obtained using this software. For further details about the software, one may visit to http://www.openbugs.net/w/FrontPage. One may also refer to a book length treatment on Bayesian analysis using OpenBUGS by Kelly and Smith [19]. We mention that in OpenBUGS software, to implement a prior distribution to a specific model, a program includes three sections namely "Model", "Data", and "Initial values". We have given the algorithm for all the three sections in Appendix. Further, the procedure to run the program in OpenBUGS is also provided.

\section{Bayesian prediction}

This section deals with Bayesian prediction of future unknown observable when it is known that $L N(\mu, \tau)$ is the underlying distribution. Bayesian approach is widely used in a variety of prediction problems including medical experiments, regression models and various industrial experiments. The two most common such problems which are frequently studied under Bayesian context are one-sample prediction and two-sample prediction.

\subsection{One-sample prediction}

Suppose that $n$ identical units whose lifetime follows $L N(\mu, \tau)$ distribution are put on a life test and a progressive Type-II censored sample $\boldsymbol{x}=\left(x_{(1)}, x_{(2)}, \ldots, x_{(m)}\right)$ of size $m$ is observed under a prescribed censoring scheme $\boldsymbol{R}=\left(R_{1}, R_{2}, \ldots, R_{m}\right)$. Recall that at the time of $j$ th failure $x_{(j)}, R_{j}$ number of live units are randomly removed from the experiment. Now, assume that $\boldsymbol{z}_{j}=\left(z_{(j 1)}, z_{(j 2)}, \ldots, z_{\left(j R_{j}\right)}\right)$ represents a vector of the ordered lifetimes of such a censored test units. Then, one-sample prediction problem involves the prediction and related inference about the censored observations $y=\left(z_{(j k)}: j=1,2, \ldots, m\right.$ and $k=$ $\left.1,2, \ldots, R_{j}\right)$. The conditional density of $y$ given the progressive Type-II censored sample $\boldsymbol{x}$ with censoring scheme $\boldsymbol{R}=\left(R_{1}, R_{2}, \ldots, R_{m}\right)$ is given by, (see also, Balakrishnan and Cramer [6])

$$
f_{1}(y \mid \boldsymbol{x}, \theta)=k\left(\begin{array}{c}
R_{j} \\
k
\end{array}\right) \frac{\left(F(y)-F\left(x_{(j)}\right)\right)^{k-1}(1-F(y))^{R_{j}-k} f(y)}{\left(1-F\left(x_{(j)}\right)\right)^{R_{j}}}, \quad y>x_{(j)} .
$$

Here, for easy reference, we denote $f(\cdot ; \theta)=f(\cdot)$ and $F(\cdot ; \theta)=F(\cdot)$. Further, observe that

$$
\begin{aligned}
\left(F(y)-F\left(x_{(j)}\right)\right)^{k-1} & =\left(\left(1-F\left(x_{(j)}\right)\right)-(1-F(y))\right)^{k-1} \\
& =\sum_{i=0}^{k-1}\left(\begin{array}{c}
k-1 \\
i
\end{array}\right)(-1)^{k-1-i}\left(1-F\left(x_{(j)}\right)\right)^{i}(1-F(y))^{k-1-i} .
\end{aligned}
$$


Thus, the conditional density given by Equation (5.1) can be re-written as

$$
f_{1}(y \mid \boldsymbol{x}, \theta)=k\left(\begin{array}{c}
R_{j} \\
k
\end{array}\right) \sum_{i=0}^{k-1}\left(\begin{array}{c}
k-1 \\
i
\end{array}\right)(-1)^{k-1-i}\left(1-F\left(x_{(j)}\right)\right)^{i-R_{j}}(1-F(y))^{R_{j}-1-i} f(y) .
$$

If one considers a prior $\pi(\theta)$ for the unknown parameter $\theta$, then the corresponding onesample posterior predictive density can be written as

$$
f_{1}^{*}(y \mid \boldsymbol{x})=\int_{\Theta} f_{1}(y \mid \boldsymbol{x}, \theta) \pi(\theta \mid \boldsymbol{x}) d \theta .
$$

Subsequently, a future unknown observable can be predicted using the corresponding one-sample posterior predictive density. More specifically, if our goal is to predict the $k$-th observation from $R_{j}$-th censored units, $j=1,2, \ldots, m$, then under the squared error loss, it is given by

$$
\hat{y}=\int_{x_{(j)}}^{\infty} y f_{1}^{*}(y \mid \boldsymbol{x}) d y
$$

Further, to compute the above expression under a prior as discussed in the Section 3, the importance sampling technique can be taken into consideration. For instance, consider the case when both $\mu$ and $\tau$ are unknown and the prior is given by Equation (3.1). Then, predictive observation $\hat{y}$ can be obtained from the following expression

$$
\begin{aligned}
\hat{y} & =\int_{-\infty}^{\infty} \int_{0}^{\infty}\left\{\int_{x_{(j)}}^{\infty} y f_{1}(y \mid \boldsymbol{x}, \mu, \tau) d y\right\} \pi(\mu, \tau \mid \boldsymbol{x}) d \tau d \mu \\
& =\int_{-\infty}^{\infty} \int_{0}^{\infty} I_{1}(\mu, \tau) \pi(\mu, \tau \mid \boldsymbol{x}) d \tau d \mu
\end{aligned}
$$

where

$$
\begin{aligned}
I_{1}(\mu, \tau)= & \int_{x_{(j)}}^{\infty} y f_{1}(y \mid \boldsymbol{x}, \mu, \tau) d y \\
= & k\left(\begin{array}{c}
R_{j} \\
k
\end{array}\right) \sum_{i=0}^{k-1}\left(\begin{array}{c}
k-1 \\
i
\end{array}\right)(-1)^{k-1-i}\left(1-F\left(x_{(j)}\right)\right)^{i-R_{j}} \\
& \quad \int_{F\left(x_{(j)}\right)}^{1} e^{\mu+\sqrt{\tau} \Phi^{-1}(v)}(1-v)^{R_{j}-1-i} d v .
\end{aligned}
$$

To compute the expression given by Equation (5.2), we generate the samples from corresponding posterior density as discussed in the previous section. Subsequently, the desired predictive estimate can be obtained as

$$
\hat{y}=\frac{\sum_{i=1}^{s} I_{1}\left(\mu_{i}, \tau_{i}\right) h\left(\mu_{i}, \tau_{i}\right)}{\sum_{i=1}^{s} h\left(\mu_{i}, \tau_{i}\right)} .
$$

In a similar way, the predictive estimates for the observation $y=\left(z_{(j k)}: j=1,2, \ldots, m\right.$ and $\left.k=1,2, \ldots, R_{j}\right)$ can be obtained under other proposed priors.

Next, we consider the problem of obtaining Bayesian predictive intervals for $y=\left(z_{(j k)}\right.$ : $j=1,2, \ldots, m$ and $\left.k=1,2, \ldots, R_{j}\right)$ observation. Notice that, under one-sample, a Bayesian predictive credible interval $(L, U)$ with degree of belief $1-\gamma$ is an interval which satisfies the following condition

$$
\int_{L}^{U} f_{1}^{*}(y \mid \boldsymbol{x}) d y=1-\gamma
$$

Here different pairs of $(L, U)$ may satisfy Equation (5.3). However, the $(1-\gamma)$ equal-tail predictive interval for $y$ can be obtained by solving the following non-linear equations for the lower bound $L$ and the upper bound $U$ :

$$
S_{1}^{*}(L \mid \boldsymbol{x})=1-\frac{\gamma}{2} \quad \text { and } \quad S_{1}^{*}(U \mid \boldsymbol{x})=\frac{\gamma}{2},
$$


where $S_{1}^{*}(\cdot \mid \boldsymbol{x})$ represents the posterior predictive survival function, and is given by

$$
S_{1}^{*}(t \mid \boldsymbol{x})=\int_{\Theta} S_{1}(t \mid \boldsymbol{x}, \theta) \pi(\theta \mid \boldsymbol{x}) d \theta .
$$

Further, the one-sample survival function $S_{1}(t \mid \boldsymbol{x}, \theta)$ can be obtained as

$$
S_{1}(t \mid \boldsymbol{x}, \theta)=\frac{P(y>t \mid \boldsymbol{x}, \theta)}{P\left(y>x_{(j)} \mid \boldsymbol{x}, \theta\right)}=\frac{\int_{t}^{\infty} f_{1}(y \mid \boldsymbol{x}, \theta) d y}{\int_{x_{(j)}}^{\infty} f_{1}(y \mid \boldsymbol{x}, \theta) d y}
$$

where

$$
\int_{t}^{\infty} f_{1}(y \mid \boldsymbol{x}, \theta) d y=k\left(\begin{array}{c}
R_{j} \\
k
\end{array}\right) \sum_{i=0}^{k-1}\left(\begin{array}{c}
k-1 \\
i
\end{array}\right)(-1)^{k-1-i}\left(1-F\left(x_{(j)}\right)\right)^{i-R_{j}} \frac{(1-F(t))^{R_{j}-i}}{R_{j}-i},
$$

and

$$
\int_{x_{(j)}}^{\infty} f_{1}(y \mid \boldsymbol{x}, \theta) d y=k\left(\begin{array}{c}
R_{j} \\
k
\end{array}\right) \sum_{i=0}^{k-1}\left(\begin{array}{c}
k-1 \\
i
\end{array}\right)(-1)^{k-1-i} \frac{1}{R_{j}-i} .
$$

Furthermore, the highest posterior density (HPD) predictive interval estimates can be obtained by using the algorithm given by Turkkan and Pham-Gia [40]. However, alternatively in case of uni-modal posterior predictive density, the HPD predictive interval estimates can also be obtained on simultaneously solving the Equations (5.3) and (5.4) for $L$ and $U$ :

$$
f_{1}^{*}(L \mid \boldsymbol{x})=f_{1}^{*}(U \mid \boldsymbol{x}) .
$$

We further mention that a numerical technique is required to solve the two equations simultaneously. We propose to use nleqslv(.) package in $\mathrm{R}$ software, and in the process, the corresponding equal-tail predictive intervals can be taken as an initial guess. We mention that, for a given parameter value and prior information, behaviour of the corresponding posterior predictive density can be observed graphically. One may also refer to Hyndman [15] for some graphical methods to observe the highest density regions.

\subsection{Two-sample prediction}

Let $\boldsymbol{x}=\left(x_{(1)}, x_{(2)}, \ldots, x_{(m)}\right)$ be an observed progressive Type-II censored sample of size $m$ corresponding to a given censoring scheme $\boldsymbol{R}=\left(R_{1}, R_{2}, \ldots, R_{m}\right)$. Further, assume that $y=\left(y_{(1)}, y_{(2)}, \ldots, y_{(M)}\right)$ be a future unobserved progressive Type-II censored sample of size $M$ from a parent sample of size $N$ corresponding to a censoring scheme $\boldsymbol{S}=\left(S_{1}, S_{2}, \ldots, S_{M}\right)$. Notice that these two samples are independently generated from a $L N(\mu, \tau)$ distribution. Then a two-sample prediction problem involves the prediction and making related inference about a future unobserved progressive Type-II censored sample $y$. We notice that the probability density function of the $k$ th order statistics of the future sample is given by

$$
f_{2}(y \mid \theta)=f_{Y_{j}}(y \mid \theta)=c_{j-1} \sum_{i=1}^{j} a_{i, j}(1-F(y))^{r_{i}-1} f(y), \quad y>0,
$$

where

$$
\begin{aligned}
& r_{j}=\sum_{i=j}^{M}\left(S_{i}+1\right)=N-\sum_{i=1}^{j-1}\left(S_{i}+1\right), \quad c_{j-1}=\prod_{i=1}^{j} r_{i}, \\
& a_{1,1}=1, \quad \text { and } \quad a_{i, j}=\prod_{k=1, k \neq i}^{j} 1 /\left(r_{k}-r_{i}\right), \quad 1 \leq i \leq j \leq M .
\end{aligned}
$$


Further, the corresponding two-sample survival function is given by

$$
S_{2}(t \mid \theta)=\int_{t}^{\infty} f_{2}(y \mid \theta) d y=c_{j-1} \sum_{i=1}^{j} a_{i, j} \frac{(1-F(t))^{r_{i}}}{r_{i}} .
$$

Under a prior $\pi(\theta)$, the two-sample posterior predictive density and the posterior predictive survival function can be obtained as

$$
f_{2}^{*}(y \mid \boldsymbol{x})=\int_{\Theta} f_{2}(y \mid \theta) \pi(\theta \mid \boldsymbol{x}) d \theta
$$

and

$$
S_{2}^{*}(t \mid \boldsymbol{x})=\int_{\Theta} S_{2}(t \mid \theta) \pi(\theta \mid \boldsymbol{x}) d \theta .
$$

Subsequently, the $j$-th predictive observation $\hat{y}_{(j)}, j=1,2, \ldots, M$, under the squared error loss can be obtained using the posterior predictive density as

$$
\hat{y(j)}=\int_{0}^{\infty} y f_{2}^{*}(y \mid \boldsymbol{x}) d y \text {. }
$$

The above expression can be computed using the importance sampling technique in a manner similar to the previous section. Furthermore, the corresponding equal-tail $100(1-\gamma) \%$ prediction interval $\left(L_{2}, U_{2}\right)$ can be obtained by solving the following non-linear equations:

$$
S_{2}^{*}(L \mid \boldsymbol{x})=1-\frac{\gamma}{2} \quad \text { and } \quad S_{2}^{*}(U \mid \boldsymbol{x})=\frac{\gamma}{2} .
$$

Finally, in a similar way, the $100(1-\gamma) \%$ HPD intervals can also be obtained using the two-sample posterior predictive density.

\section{Data analysis and simulation study}

\subsection{Data analysis}

In this section, we study a real data set to illustrate the methods develop for one- and two-sample prediction problems. In the process, we analyze a data set as given in Lawless [24]. This data set represents the number of million revolutions before failure for 23 ball bearings. The corresponding observations are:

$17.88,28.92,33.00,41.52,42.12,45.60,48.40,51.84,51.96,54.12$, $55.56,67.80,68.64,68.64,68.88,84.12,93.12,98.64,105.12,105.84$, $127.92,128.04,173.40$

Singh et al. [36] showed that $L N(\mu, \tau)$ distribution fits this data set reasonably well with corresponding MLEs given by $\hat{\mu}=4.15038$ and $\hat{\tau}=0.27215$. Authors further generated a progressive Type-II censored sample of size $m=12$ from the given data set with respect to the censoring scheme $R=\left(0^{* 11}, 11\right)$. This censoring scheme represents that 0 number of units will be removed at the time of the first 11 failures and 11 live units will be removed at 12th failure. Therefore, the corresponding progressive Type-II censored data become the first 12 observations of the given data set that is the observations up to 67.80 lifetime. We consider both the complete and the progressive Type-II censored data sets for further Bayesian estimation and prediction analysis. We take into account non-informative priors for all the three cases discussed in Section 3. In the process for convenience, under case (i) we consider the true value of $\tau$ equal to the maximum likelihood estimated value, that is $\tau=0.27215$, and similarly in case of (ii) the value of $\mu$ as $\mu=4.15038$. First we obtain Bayes estimates using importance sampling technique and OpenBUGS software. We also compute $95 \%$ Bayesian confidence interval estimates using the method of Chen and Shao [11] and OpenBUGS software. All the estimated values are reported in Table 1. We mention that $\mathrm{R}$ statistical programming language is taken into consideration and the reported observations are based on 1000 Monte Carlo simulations. From tabulated values, 
Table 1. Bayes and 95\% Bayesian credible interval estimates of $\mu$ and $\tau$ for given data

\begin{tabular}{|c|c|c|c|c|c|}
\hline \multirow{2}{*}{\multicolumn{2}{|c|}{ Case Parameter }} & \multicolumn{2}{|c|}{ Complete data } & \multicolumn{2}{|c|}{ Progressive Type-II censored data } \\
\hline & & MCMC & OpenBUGS & MCMC & OpenBUGS \\
\hline (i) & $\mu$ & $4.1515(3.9342,4.3628)$ & $4.1510(3.9410,4.3687)$ & $4.1701(3.9461,4.3125)$ & $4.1676(3.9396,4.3970)$ \\
\hline (ii) & $\tau$ & $0.2980(0.1626,0.5252)$ & $0.2991(0.1661,0.540)$ & $0.3678(0.1539,0.8352)$ & $0.3677(0.1552,0.8226)$ \\
\hline \multirow[t]{2}{*}{ (iii) } & $\mu$ & $4.1502(3.9204,4.3717)$ & $4.1507(3.9254,4.3773)$ & $4.2502(3.9643,4.4290)$ & $4.2301(3.9369,4.6449)$ \\
\hline & $\tau$ & $0.3138(0.1689,0.5716)$ & $0.3199(0.1707,0.5629)$ & $0.5298(0.1719,0.7945)$ & $0.4626(0.1654,1.1994)$ \\
\hline
\end{tabular}

Table 2. One sample Bayesian prediction for progressive Type-II censored data

\begin{tabular}{ccccccccccc}
\hline & & \multicolumn{4}{c}{ Equal-tail interval } & & \multicolumn{3}{c}{ HPD interval } \\
\cline { 10 - 11 } \cline { 8 - 10 } Case & $j$ & $k$ & $\hat{y}$ & $L$ & $U$ & AIL & & $L$ & $U$ & AIL \\
\hline (i) & $m$ & 1 & 71.40 & 67.89 & 81.06 & 13.17 & & 67.80 & 78.52 & 10.72 \\
& 2 & 75.30 & 67.01 & 88.65 & 21.64 & & 67.96 & 85.66 & 17.70 \\
& 3 & 79.56 & 70.22 & 96.14 & 25.92 & & 68.98 & 92.90 & 23.92 \\
& 4 & 84.28 & 72.26 & 104.11 & 31.85 & & 70.69 & 100.63 & 29.96 \\
& 5 & 89.62 & 74.80 & 113.00 & 38.20 & & 72.93 & 109.26 & 36.33 \\
& 6 & 95.79 & 77.87 & 123.33 & 45.46 & & 75.67 & 119.16 & 43.49 \\
(ii) & 1 & 71.94 & 67.90 & 83.77 & 15.87 & & 67.80 & 80.50 & 12.70 \\
& 2 & 76.47 & 66.95 & 93.93 & 26.98 & & 67.92 & 89.42 & 21.50 \\
& 3 & 81.48 & 70.38 & 104.57 & 34.19 & & 68.82 & 98.95 & 30.13 \\
& 4 & 87.12 & 72.52 & 116.54 & 44.02 & & 70.33 & 109.57 & 39.24 \\
& 5 & 93.58 & 75.14 & 130.55 & 55.41 & & 72.29 & 121.82 & 49.53 \\
& 6 & 101.17 & 78.26 & 147.58 & 69.32 & & 74.64 & 136.48 & 61.84 \\
(iii) & 1 & 72.11 & 67.90 & 84.49 & 16.59 & & 67.80 & 81.13 & 13.33 \\
& 2 & 76.83 & 67.02 & 94.95 & 27.93 & & 67.93 & 90.48 & 22.55 \\
& 3 & 82.08 & 70.39 & 105.80 & 35.41 & & 68.85 & 100.41 & 31.56 \\
& 4 & 88.00 & 72.12 & 117.89 & 45.77 & & 70.38 & 111.47 & 41.09 \\
& 5 & 94.82 & 75.08 & 131.96 & 56.88 & & 72.36 & 124.23 & 51.87 \\
& 6 & 102.86 & 77.59 & 149.04 & 71.45 & & 74.73 & 139.53 & 64.80 \\
\hline
\end{tabular}

it is seen that estimated values obtained using OpenBUGS and importance sampling technique are very close.

Next, we consider the progressive Type-II censored data as informative sample and illustrate one-sample prediction problem. Recall that the progressive Type-II censored data has first 12 observations of the given ball bearings data set. Under the one-sample prediction problem, we predict the remaining first 6 censored observation, corresponding 95\% equal-tail and HPD predictive intervals. All the estimated values are reported in Table 2. From tabulated values, it is seen that with a higher value of $k$ the average interval length (AIL) of predictive bounds tend to increase. It is also observed that the reported predictive bound contain the true lifetime of the given ball bearing data set. This holds true under all the three cases and for both the equal-tail and HPD predictive intervals. Tabulated values also suggest that prediction bounds of future observations are likely to be small for the cases where some partial information about the parameters is available. As it is seen that AIL is smaller under case (i) followed by case (ii) and case (iii). Finally, it is observed that the AIL of HPD predictive intervals is smaller than that of AIL of equal tail predictive intervals and this holds true for all the three cases.

Now, we illustrate the two-sample prediction problem based on observed progressive Type-II censored data. We predict about the future sample of size $N=23$ independent 
Table 3. Two-sample Bayesian prediction for progressive Type-II censored data

\begin{tabular}{|c|c|c|c|c|c|c|c|c|c|c|c|c|c|c|c|}
\hline \multirow[b]{3}{*}{ Case } & \multirow[b]{3}{*}{$j$} & \multicolumn{7}{|c|}{$S=\left(0^{* 11}, 11\right)$} & \multicolumn{7}{|c|}{$S=\left(11,0^{* 11}\right)$} \\
\hline & & \multirow[b]{2}{*}{$\hat{y}_{j}$} & \multicolumn{3}{|c|}{ Equal-tail interval } & \multicolumn{3}{|c|}{ HPD interval } & \multirow[b]{2}{*}{$\hat{y}_{j}$} & \multicolumn{3}{|c|}{ Equal-tail interval } & \multicolumn{3}{|c|}{ HPD interval } \\
\hline & & & $L$ & $U$ & AIL & $L$ & $U$ & AIL & & $L$ & $U$ & AIL & $L$ & $U$ & AIL \\
\hline \multirow[t]{6}{*}{ (i) } & 1 & 23.98 & 12.42 & 37.86 & 25.44 & 11.68 & 36.90 & 25.22 & 23.98 & 12.42 & 37.85 & 25.43 & 11.68 & 36.90 & 25.22 \\
\hline & 2 & 29.87 & 18.22 & 43.78 & 25.56 & 17.52 & 42.87 & 25.35 & 33.49 & 19.61 & 51.52 & 31.91 & 18.43 & 49.82 & 31.39 \\
\hline & 3 & 34.17 & 22.10 & 48.50 & 26.40 & 21.40 & 47.60 & 26.20 & 40.49 & 24.90 & 60.67 & 35.77 & 23.60 & 58.79 & 35.19 \\
\hline & 4 & 37.86 & 25.24 & 52.75 & 27.51 & 24.52 & 51.83 & 27.31 & 46.79 & 29.51 & 69.09 & 39.58 & 28.08 & 67.03 & 38.95 \\
\hline & 5 & 41.24 & 28.02 & 56.76 & 28.74 & 27.27 & 55.83 & 28.56 & 52.98 & 33.88 & 77.65 & 43.77 & 32.29 & 75.36 & 43.07 \\
\hline & 6 & 44.46 & 30.58 & 60.68 & 30.10 & 29.82 & 59.72 & 29.90 & 59.42 & 38.26 & 86.88 & 48.62 & 36.46 & 84.28 & 47.82 \\
\hline \multirow[t]{6}{*}{ (ii) } & 1 & 21.82 & 7.63 & 37.17 & 29.54 & 7.12 & 36.57 & 29.45 & 21.81 & 7.64 & 37.16 & 29.52 & 7.12 & 36.56 & 29.44 \\
\hline & 2 & 27.62 & 12.69 & 42.53 & 29.84 & 12.59 & 42.43 & 29.84 & 31.31 & 14.41 & 49.74 & 35.33 & 13.76 & 48.98 & 35.22 \\
\hline & 3 & 31.97 & 16.67 & 46.79 & 30.12 & 16.74 & 46.86 & 30.12 & 38.57 & 20.26 & 58.65 & 38.39 & 19.54 & 57.81 & 38.27 \\
\hline & 4 & 35.77 & 20.22 & 50.65 & 30.43 & 20.36 & 50.78 & 30.42 & 45.27 & 25.82 & 67.40 & 41.58 & 24.84 & 66.19 & 41.35 \\
\hline & 5 & 39.30 & 23.58 & 54.39 & 30.81 & 23.70 & 54.51 & 30.81 & 52.02 & 31.34 & 76.98 & 45.64 & 29.92 & 75.11 & 45.19 \\
\hline & 6 & 42.71 & 26.84 & 58.16 & 31.32 & 26.88 & 58.20 & 31.32 & 59.19 & 36.97 & 88.14 & 51.17 & 34.96 & 85.24 & 50.28 \\
\hline \multirow[t]{6}{*}{ (iii) } & 1 & 20.97 & 7.84 & 36.04 & 28.20 & 7.13 & 35.07 & 27.94 & 20.95 & 7.91 & 36.04 & 28.13 & 7.20 & 35.05 & 27.85 \\
\hline & 2 & 26.73 & 12.71 & 41.74 & 29.03 & 12.26 & 41.15 & 28.89 & 30.44 & 14.33 & 49.53 & 35.20 & 13.30 & 48.06 & 34.76 \\
\hline & 3 & 31.08 & 16.42 & 46.39 & 29.97 & 16.07 & 45.92 & 29.85 & 37.77 & 19.74 & 59.31 & 39.57 & 18.57 & 57.64 & 39.07 \\
\hline & 4 & 34.91 & 19.68 & 50.68 & 30.00 & 19.36 & 50.24 & 30.88 & 44.60 & 24.82 & 68.95 & 44.13 & 23.42 & 66.88 & 43.46 \\
\hline & 5 & 38.48 & 22.71 & 54.86 & 32.15 & 22.38 & 54.40 & 32.02 & 51.54 & 29.89 & 79.42 & 49.53 & 28.12 & 76.73 & 48.61 \\
\hline & 6 & 41.94 & 25.64 & 59.08 & 33.44 & 25.26 & 58.55 & 33.29 & 58.96 & 35.08 & 91.46 & 56.38 & 32.83 & 87.85 & 55.02 \\
\hline
\end{tabular}

of the observed progressive Type-II censored data. For convenience, we take effective sample size $M=m$ under the two future censoring schemes such as $\boldsymbol{S}=\left(0^{* 11}, 11\right)$ and $\boldsymbol{S}=\left(11,0^{* 11}\right)$. The estimated values are reported in Table 3 . From tabulated values, it is seen that prediction interval using the censoring scheme $\boldsymbol{S}=\left(11,0^{* 11}\right)$ are in general wider than the corresponding prediction interval obtained using the censoring scheme $\boldsymbol{S}=\left(0^{* 11}, 11\right)$. This observation holds true under all the three cases and for both equaltail and HPD predictive intervals. Likewise under one-sample prediction, here also we observe that AIL is smaller under case (i) followed by case (ii) and case (iii). However, the AIL of equal-tail and HPD predictive intervals are almost same, as was not found under one-sample prediction problem.

\subsection{Simulation study}

In this section, a Monte Carlo simulation study is performed to investigate the behaviour of $95 \%$ Bayesian prediction bound under both one- and two-sample prediction problems. We consider the case (iii) and simulate progressive Type-II censored samples from $\operatorname{LN}(0,1)$ distribution for different combinations of $n, m$ and censoring schemes. Various prediction estimates and prediction intervals of censored observations are computed based on 5000 replications of progressive Type-II censored samples for a given censoring scheme. The $\mathrm{R}$ statistical software is used for all computations. In particular, we used nleqslv $(\cdot)$ package of this software. Under one-sample prediction problem, we obtain $95 \%$ equal-tail and HPD predictive bounds, corresponding AILs under non-informative and informative prior situations. All the estimated values are reported in Table 4. We mention that in the table, the non-informative prior are denoted as Prior I and informative prior as Prior II. In case of informative prior, hyper-parameters are assigned values like $a=0.01, b=1$, $p_{2}=3$, and $q_{2}=4$. These values are selected such that the prior means remain close to the true parameter values. From tabulated values, it is seen that a higher value of $k$ and $m$ lead to wider prediction bound. Further, the AIL are larger in case when units 
Table 4. One-sample Bayesian prediction interval and average interval lengths

\begin{tabular}{|c|c|c|c|c|c|c|c|c|c|c|c|c|c|c|c|c|}
\hline \multirow[b]{3}{*}{$n$} & \multirow[b]{3}{*}{$m$} & \multirow[b]{3}{*}{$R$} & \multirow[b]{3}{*}{$j$} & \multirow[b]{3}{*}{$k$} & \multicolumn{6}{|c|}{ Prior I } & \multicolumn{6}{|c|}{ Prior II } \\
\hline & & & & & \multicolumn{3}{|c|}{ Equal-tail interval } & \multicolumn{3}{|c|}{ HPD interval } & \multicolumn{3}{|c|}{ Equal-tail interval } & \multicolumn{3}{|c|}{ HPD interval } \\
\hline & & & & & $L$ & $U$ & AIL & $L$ & $U$ & AIL & $L$ & $U$ & AIL & $L$ & $U$ & AIL \\
\hline \multirow[t]{12}{*}{15} & 6 & $\left(9,0^{* 5}\right)$ & 1 & 1 & 0.215 & 0.993 & 0.778 & 198 & 0.791 & 0.593 & 0.216 & 0.895 & 0.679 & 0.202 & 0.742 & 0.540 \\
\hline & & & & 2 & 0.191 & 1.622 & 1.431 & 0.214 & 1.281 & 1.067 & 0.196 & 1.302 & 1.106 & 0.218 & 1.106 & 0.888 \\
\hline & & & & 3 & 0.311 & 2.508 & 2.197 & 0.239 & 1.906 & 1.667 & 0.317 & 1.770 & 1.453 & 0.252 & 1.498 & 1.246 \\
\hline & & $\left(0^{* 5}, 9\right)$ & 6 & 1 & 0.682 & 1.366 & 0.684 & 0.738 & 1.435 & 0.697 & 0.759 & 1.304 & 0.545 & 0.740 & 1.150 & 0.410 \\
\hline & & & & 2 & 0.661 & 2.077 & 1.416 & 0.680 & 1.694 & 1.014 & 0.741 & 1.721 & 0.980 & 0.764 & 1.554 & 0.790 \\
\hline & & & & 3 & 0.747 & 3.161 & 2.414 & 0.693 & 2.435 & 1.742 & 0.829 & 2.221 & 1.392 & 0.778 & 1.937 & 1.159 \\
\hline & 9 & $\left(6,0^{* 8}\right)$ & 1 & 1 & 0.212 & 1.218 & 1.006 & 0.205 & 1.008 & 0.803 & 0.194 & 1.089 & 0.895 & 0.195 & 0.928 & 0.733 \\
\hline & & & & 2 & 0.174 & 2.105 & 1.931 & 0.212 & 1.681 & 1.469 & 0.171 & 1.762 & 1.591 & 0.181 & 1.559 & 1.378 \\
\hline & & & & 3 & 0.367 & 3.533 & 3.166 & 0.255 & 2.727 & 2.472 & 0.347 & 2.731 & 2.384 & 0.248 & 2.238 & 1.990 \\
\hline & & $\left(0^{* 8}, 6\right)$ & 9 & 1 & 1.214 & 2.601 & 1.387 & 1.236 & 2.300 & 1.064 & 1.230 & 2.171 & 0.941 & 1.253 & 1.983 & 0.730 \\
\hline & & & & 2 & 1.193 & 4.193 & 3.000 & 1.212 & 3.399 & 2.187 & 1.204 & 3.019 & 1.815 & 1.229 & 2.635 & 1.406 \\
\hline & & & & 3 & 1.356 & 6.931 & 5.575 & 1.238 & 5.340 & 4.102 & 1.361 & 4.232 & 2.871 & 1.263 & 3.601 & 2.338 \\
\hline \multirow[t]{12}{*}{20} & 8 & $\left(12,0^{* 7}\right)$ & 1 & 1 & 0.180 & 0.727 & 0.547 & 0.173 & 0.608 & 0.435 & 0.182 & 0.698 & 0.516 & 0.178 & 0.598 & 0.420 \\
\hline & & & & 2 & 0.168 & 1.061 & 0.893 & 0.184 & 0.990 & 0.806 & 0.183 & 0.968 & 0.785 & 0.184 & 0.840 & 0.656 \\
\hline & & & & 3 & 0.255 & 1.447 & 1.192 & 0.205 & 1.207 & 1.002 & 0.262 & 1.250 & 0.988 & 0.215 & 1.089 & 0.874 \\
\hline & & $\left(0^{* 7}, 12\right)$ & 8 & 1 & 0.737 & 1.173 & 0.436 & 0.751 & 1.108 & 0.357 & 0.762 & 1.127 & 0.365 & 0.761 & 1.051 & 0.290 \\
\hline & & & & 2 & 0.724 & 1.516 & 0.792 & 0.737 & 1.351 & 0.614 & 0.744 & 1.375 & 0.631 & 0.762 & 1.263 & 0.501 \\
\hline & & & & 3 & 0.788 & 1.927 & 1.139 & 0.751 & 1.687 & 0.936 & 0.812 & 1.643 & 0.831 & 0.778 & 1.499 & 0.720 \\
\hline & 10 & $\left(10,0^{* 9}\right)$ & 1 & 1 & 0.180 & 0.801 & 0.621 & 0.175 & 0.675 & 0.500 & 0.178 & 0.760 & 0.582 & 0.178 & 0.668 & 0.490 \\
\hline & & & & 2 & 0.171 & 1.185 & 1.014 & 0.182 & 1.003 & 0.821 & 0.192 & 1.080 & 0.888 & 0.181 & 0.942 & 0.761 \\
\hline & & & & 3 & 0.275 & 1.640 & 1.365 & 0.215 & 1.380 & 1.165 & 0.276 & 1.430 & 1.154 & 0.220 & 1.243 & 1.023 \\
\hline & & $\left(0^{* 9}, 10\right)$ & 10 & 1 & 0.989 & 1.549 & 0.560 & 0.978 & 1.422 & 0.444 & 0.983 & 1.453 & 0.470 & 0.982 & 1.356 & 0.374 \\
\hline & & & & 2 & 0.970 & 1.986 & 1.016 & 0.989 & 1.787 & 0.798 & 0.961 & 1.785 & 0.824 & 0.983 & 1.638 & 0.655 \\
\hline & & & & 3 & 1.061 & 2.515 & 1.454 & 1.009 & 2.229 & 1.220 & 1.050 & 2.160 & 1.110 & 1.005 & 1.966 & 0.961 \\
\hline \multirow[t]{12}{*}{25} & 10 & $\left(15,0^{* 9}\right)$ & 1 & 1 & 0.163 & 0.603 & 0.440 & 0.156 & 0.515 & 0.359 & 0.167 & 0.598 & 0.431 & 0.155 & 0.513 & 0.358 \\
\hline & & & & 2 & 0.160 & 0.833 & 0.673 & 0.164 & 0.722 & 0.558 & 0.182 & 0.800 & 0.618 & 0.170 & 0.706 & 0.536 \\
\hline & & & & 3 & 0.227 & 1.071 & 0.844 & 0.188 & 0.930 & 0.742 & 0.237 & 0.998 & 0.761 & 0.199 & 0.888 & 0.689 \\
\hline & & $\left(0^{* 9}, 15\right)$ & 10 & 1 & 0.755 & 1.033 & 0.278 & 0.752 & 0.985 & 0.233 & 0.765 & 1.031 & 0.266 & 0.761 & 0.971 & 0.210 \\
\hline & & & & 2 & 0.744 & 1.219 & 0.475 & 0.755 & 1.136 & 0.381 & 0.750 & 1.199 & 0.449 & 0.765 & 1.126 & 0.361 \\
\hline & & & & 3 & 0.794 & 1.418 & 0.624 & 0.768 & 1.311 & 0.543 & 0.804 & 1.372 & 0.568 & 0.779 & 1.283 & 0.504 \\
\hline & 15 & $\left(10,0^{* 14}\right)$ & 1 & 1 & 0.162 & 0.721 & 0.559 & 0.159 & 0.632 & 0.473 & 0.167 & 0.726 & 0.559 & 0.160 & 0.635 & 0.475 \\
\hline & & & & 2 & 0.165 & 1.047 & 0.882 & 0.165 & 0.903 & 0.738 & 0.192 & 1.024 & 0.832 & 0.173 & 0.898 & 0.725 \\
\hline & & & & 3 & 0.257 & 1.417 & 1.160 & 0.202 & 1.225 & 1.023 & 0.271 & 1.347 & 1.076 & 0.218 & 1.188 & 0.970 \\
\hline & & $\left(0^{* 14}, 10\right)$ & 15 & 1 & 1.245 & 1.786 & 0.541 & 1.248 & 1.677 & 0.429 & 1.262 & 1.751 & 0.489 & 1.259 & 1.641 & 0.382 \\
\hline & & & & 2 & 1.220 & 2.181 & 0.961 & 1.245 & 2.008 & 0.763 & 1.236 & 2.091 & 0.855 & 1.262 & 1.945 & 0.683 \\
\hline & & & & 3 & 1.323 & 2.637 & 1.314 & 1.270 & 2.404 & 1.134 & 1.336 & 2.471 & 1.135 & 1.288 & 2.283 & 0.995 \\
\hline
\end{tabular}

are censored at the first stage than that of when units are censored at the last stage. It is also seen that the predictive intervals under Prior II have smaller AILs than those of Prior I. Furthermore, the AILs of HPD predictive intervals are smaller than those of equal-tail predictive intervals. Next, we present the corresponding estimated values under two-sample prediction problem in Table 5 . We mention that the future sample which is independent of the informative sample is considered of size $N=n$ and $M=m$. Further, the future censoring scheme $\boldsymbol{S}=\left(S_{1}, S_{2}, \ldots, S_{M}\right)$ is also taken to be the same as the censoring scheme $\boldsymbol{R}=\left(R_{1}, R_{2}, \ldots, R_{m}\right)$ of the informative sample. From tabulated values, similar results likewise one-sample prediction can be seen. 
Table 5. Two-sample Bayesian prediction interval and average interval lengths

\begin{tabular}{|c|c|c|c|c|c|c|c|c|c|c|c|c|c|c|c|}
\hline \multirow{3}{*}{$\begin{array}{l}n \\
N\end{array}$} & \multirow{3}{*}{$\begin{array}{l}m \\
M\end{array}$} & \multirow{2}{*}{\multicolumn{2}{|c|}{$R$}} & \multicolumn{6}{|c|}{ Prior I } & \multicolumn{6}{|c|}{ Prior II } \\
\hline & & & & \multicolumn{3}{|c|}{ Equal-tail interval } & \multicolumn{3}{|c|}{ HPD interval } & \multicolumn{3}{|c|}{ Equal-tail interval } & \multicolumn{3}{|c|}{ HPD interval } \\
\hline & & $S$ & $k$ & $L$ & $U$ & AIL & $L$ & $U$ & AIL & $L$ & $U$ & AIL & $L$ & $U$ & AIL \\
\hline \multirow[t]{12}{*}{15} & \multirow[t]{6}{*}{6} & \multirow[t]{3}{*}{$\left(9,0^{* 5}\right)$} & 1 & 0.034 & 0.639 & 0.605 & 0.020 & 0.600 & 0.580 & 0.051 & 0.633 & 0.582 & 0.025 & 0.552 & 0.527 \\
\hline & & & 2 & 0.107 & 1.767 & 1.660 & 0.043 & 1.356 & 1.313 & 0.140 & 1.458 & 1.318 & 0.076 & 1.220 & 1.144 \\
\hline & & & 3 & 0.209 & 3.608 & 3.399 & 0.088 & 2.593 & 2.505 & 0.246 & 2.420 & 2.174 & 0.143 & 1.986 & 1.843 \\
\hline & & \multirow[t]{3}{*}{$\left(0^{* 5}, 9\right)$} & 1 & 0.047 & 0.572 & 0.525 & 0.030 & 0.550 & 0.520 & 0.052 & 0.550 & 0.498 & 0.029 & 0.494 & 0.465 \\
\hline & & & 2 & 0.087 & 0.731 & 0.644 & 0.075 & 0.690 & 0.615 & 0.111 & 0.732 & 0.621 & 0.080 & 0.669 & 0.589 \\
\hline & & & 3 & 0.128 & 0.907 & 0.779 & 0.105 & 0.835 & 0.730 & 0.165 & 0.912 & 0.747 & 0.128 & 0.835 & 0.707 \\
\hline & \multirow[t]{6}{*}{9} & \multirow[t]{3}{*}{$\left(6,0^{* 8}\right)$} & 1 & 0.034 & 0.597 & 0.563 & 0.011 & 0.511 & 0.500 & 0.045 & 0.571 & 0.526 & 0.022 & 0.499 & 0.477 \\
\hline & & & 2 & 0.093 & 1.073 & 0.980 & 0.047 & 0.915 & 0.868 & 0.111 & 0.993 & 0.882 & 0.068 & 0.864 & 0.796 \\
\hline & & & 3 & 0.163 & 1.601 & 1.438 & 0.092 & 1.347 & 1.255 & 0.182 & 1.421 & 1.239 & 0.120 & 1.230 & 1.110 \\
\hline & & \multirow[t]{3}{*}{$\left(0^{* 8}, 6\right)$} & 1 & 0.033 & 0.540 & 0.507 & 0.022 & 0.522 & 0.500 & 0.050 & 0.550 & 0.500 & 0.026 & 0.491 & 0.465 \\
\hline & & & 2 & 0.075 & 0.741 & 0.666 & 0.051 & 0.668 & 0.617 & 0.106 & 0.731 & 0.625 & 0.075 & 0.667 & 0.592 \\
\hline & & & 3 & 0.121 & 0.957 & 0.836 & 0.083 & 0.853 & 0.770 & 0.159 & 0.907 & 0.748 & 0.122 & 0.832 & 0.710 \\
\hline \multirow[t]{12}{*}{20} & \multirow[t]{6}{*}{8} & \multirow[t]{3}{*}{$\left(12,0^{* 7}\right)$} & 1 & 0.032 & 0.503 & 0.471 & 0.033 & 0.483 & 0.450 & 0.045 & 0.507 & 0.462 & 0.024 & 0.447 & 0.423 \\
\hline & & & 2 & 0.095 & 1.144 & 1.049 & 0.048 & 0.950 & 0.902 & 0.118 & 1.056 & 0.938 & 0.071 & 0.908 & 0.837 \\
\hline & & & 3 & 0.172 & 1.871 & 1.699 & 0.094 & 1.518 & 1.424 & 0.199 & 1.585 & 1.386 & 0.129 & 1.355 & 1.226 \\
\hline & & \multirow[t]{3}{*}{$\left(0^{* 7}, 12\right)$} & 1 & 0.039 & 0.403 & 0.364 & 0.030 & 0.383 & 0.353 & 0.048 & 0.431 & 0.383 & 0.031 & 0.395 & 0.364 \\
\hline & & & 2 & 0.077 & 0.515 & 0.438 & 0.063 & 0.482 & 0.419 & 0.098 & 0.550 & 0.452 & 0.079 & 0.5 & 0.437 \\
\hline & & & 3 & 0.115 & 0.624 & 0.509 & 0.097 & 0.586 & 0.489 & 0.143 & 0.659 & 0.516 & 0.121 & 0.623 & 0.502 \\
\hline & \multirow[t]{6}{*}{10} & \multirow[t]{3}{*}{$\left(10,0^{* 9}\right)$} & 1 & 0.033 & 0.498 & 0.465 & 0.014 & 0.433 & 0.419 & 0.045 & 0.496 & 0.451 & 0.024 & 0.439 & 0.415 \\
\hline & & & 2 & 0.090 & 0.933 & 0.843 & 0.050 & 0.804 & 0.754 & 0.111 & 0.887 & 0.776 & 0.072 & 0.780 & 0.708 \\
\hline & & & 3 & 0.156 & 1.364 & 1.208 & 0.095 & 1.168 & 1.073 & 0.180 & 1.242 & 1.062 & 0.125 & 1.092 & 0.967 \\
\hline & & $\left(0^{* 9}, 10\right)$ & 1 & 0.045 & 0.423 & 0.378 & 0.031 & 0.384 & 0.353 & 0.049 & 0.444 & 0.395 & 0.031 & 0.406 & 0.375 \\
\hline & & & 2 & 0.087 & 0.541 & 0.454 & 0.071 & 0.505 & 0.434 & 0.099 & 0.568 & 0.469 & 0.078 & 0.531 & 0.453 \\
\hline & & & 3 & 0.127 & 0.651 & 0.524 & 0.108 & 0.612 & 0.504 & 0.144 & 0.679 & 0.535 & 0.121 & 0.641 & 0.520 \\
\hline 20 & 10 & $\left(15,0^{* 9}\right)$ & 1 & 0.032 & 0.433 & 0.401 & 0.015 & 0.380 & 0.365 & 0.045 & 0.445 & 0.400 & 0.026 & 0.397 & 0.371 \\
\hline & & & 2 & 0.089 & 0.888 & 0.799 & 0.050 & 0.763 & 0.713 & 0.111 & 0.860 & 0.749 & 0.073 & 0.756 & 0.683 \\
\hline & & & 3 & 0.155 & 1.315 & 1.160 & 0.097 & 1.124 & 1.027 & 0.181 & 1.214 & 1.033 & 0.128 & 1.068 & 0.940 \\
\hline & & $\left(0^{* 9}, 15\right)$ & 1 & 0.043 & 0.335 & 0.292 & 0.035 & 0.310 & 0.275 & 0.046 & 0.360 & 0.314 & 0.033 & 0.334 & 0.301 \\
\hline & & & 2 & 0.080 & 0.416 & 0.336 & 0.070 & 0.395 & 0.325 & 0.091 & 0.449 & 0.358 & 0.077 & 0.427 & 0.350 \\
\hline & & & 3 & 0.112 & 0.486 & 0.374 & 0.101 & 0.466 & 0.365 & 0.130 & 0.527 & 0.397 & 0.116 & 0.505 & 0.389 \\
\hline & 15 & $\left(10,0^{* 14}\right)$ & 1 & 0.032 & 0.400 & 0.368 & 0.015 & 0.354 & 0.339 & 0.043 & 0.422 & 0.379 & 0.025 & 0.378 & 0.353 \\
\hline & & & 2 & 0.078 & 0.638 & 0.560 & 0.050 & 0.570 & 0.520 & 0.098 & 0.655 & 0.557 & 0.070 & 0.593 & 0.523 \\
\hline & & & 3 & 0.125 & 0.843 & 0.718 & 0.089 & 0.758 & 0.669 & 0.151 & 0.849 & 0.698 & 0.116 & 0.773 & 0.657 \\
\hline & & $\left(0^{* 14}, 10\right)$ & 1 & 0.037 & 0.361 & 0.324 & 0.026 & 0.330 & 0.304 & 0.045 & 0.381 & 0.336 & 0.030 & 0.350 & 0.320 \\
\hline & & & 2 & 0.074 & 0.456 & 0.382 & 0.061 & 0.436 & 0.375 & 0.090 & 0.480 & 0.390 & 0.074 & 0.452 & 0.378 \\
\hline & & & 3 & 0.108 & 0.539 & 0.431 & 0.091 & 0.509 & 0.418 & 0.129 & 0.566 & 0.437 & 0.112 & 0.537 & 0.425 \\
\hline
\end{tabular}

\section{Conclusion}

In this paper, we have considered Bayesian estimation and prediction for $L N(\mu, \tau)$ distribution under progressive Type-II censoring. We considered three cases when (i) $\mu$ is unknown, (ii) $\tau$ is unknown and (iii) $\mu$ and $\tau$ both are unknown. We proposed non-informative and informative priors for each case and obtained Bayes estimates under squared error loss function. Importance sampling technique and OpenBUGS software were taken into consideration for the computation of Bayes estimates. We also reported the associated Bayesian credible interval estimates. We further analyzed a real data set to illustrate all the cases and found that the performance of OpenBUGS software appreciable. Next, under the setup of one- and two-sample Bayesian predictions, we obtained Bayes estimates, corresponding equal-tail and HPD predictive interval estimates based on progressive Type-II censored samples. It can be observed that $95 \%$ Bayesian prediction bound under one-sample prediction contains the true observations of the complete data 
set. We also observed that prediction intervals tend to be smaller for the cases where some partial information on the unknown parameters are available. It can also be observed that HPD predictive intervals are of smaller interval length as compared to equal-tail predictive intervals. However, for real data set under two-sample prediction framework, both the equal-tail and HPD predictive intervals are found of almost same average interval length. Our simulation study revealed that, under proper prior information, the lengths of various prediction intervals tend to be smaller compared to the situation of non-informative prior distributions. Finally, we mention that the proposed terminology for Bayesian prediction can be used for other lifetime models as well.

Acknowledgment. The authors are grateful to a reviewer and an Area Editor for providing encouraging comments that led to significant improvement in the manuscript. They also thank the Editor for helpful comments. Yogesh Mani Tripathi gratefully acknowledges the partial financial support for this research work under a grant EMR/2016/001401 Science and Engineering Research Board, India.

\section{Appendix}

For Bayesian analysis in OpenBUGS write three programs in different files, say "Model", "Data" and "Initial values" as given below

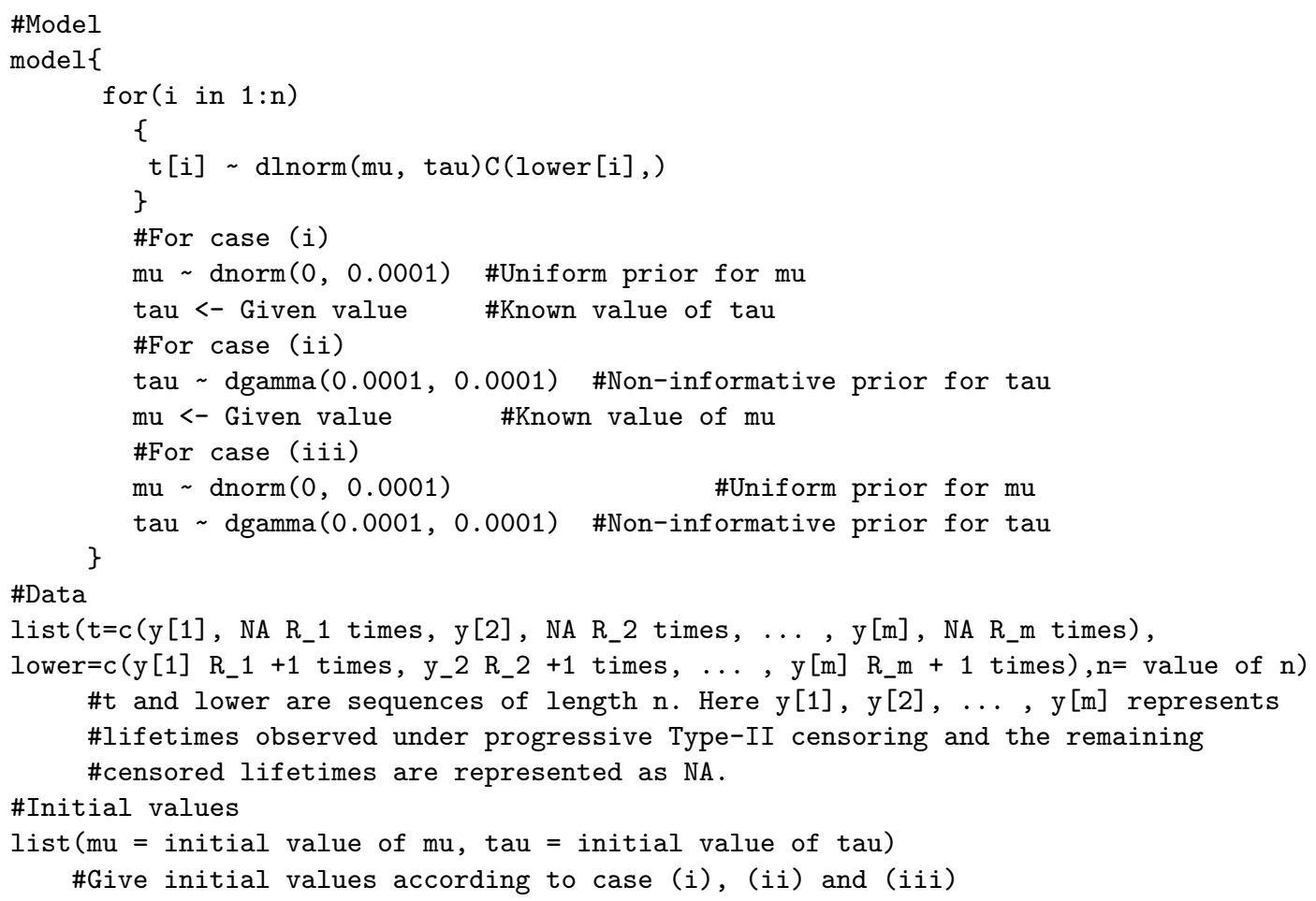

After writing all the programs follow the following procedure

(1) To initialize a model in OpenBUGS software: First open all the written program named "Model", "Data", and "Initial values". Now goto Tool bar and select open Model $\rightarrow$ Specification. It will show a dialogue box named "Specification Tool". First Double-click (highlight) the file "Model" and click "check model" in the dialogue box. A message at the bottom left-hand corner will display "model is syntactically correct" to ensure that the written program is correct. Next highlight the file "Data" and then click "load data" in the same dialogue box. For successfully loaded data a message "data loaded" will display. Next click "compile" in the dialogue box to compile the data for which a message "model compiled" will display. 
Finally, double-click the file "Initial values" and click "load inits" in the dialogue box to initialize the values. For successfully initialized case a message "model is initialized" will display. Note that if one did not initialize all the parameters, then further "gen inits" can be clicked in the dialogue box.

(2) Further to draw MCMC samples: First go to Tool bar: open Inference $\rightarrow$ Samples. A dialogue box named "Sample Monitor Tool" will appear. In the dialogue box type the parameters of interest in the "node" box and click "set". For an example we will track "mu", "tau" and both respectively in cases (i), (ii) and (iii). Next again go to Tool bar: open Model $\rightarrow$ Update. A dialogue box named "Update Tool" will appear in which type the number of posterior samples you want to generate in the "updates" box and click "update" box and see it runs in the "iteration" box. Now again go back to the "Sample Monitor Tool" and type "*" in the "node" box. Finally the Bayesian analysis of the desired parameters can be seen from the "stats" box.

Note that in "Sample Monitor Tool" dialogue box one may also select the number of burnin samples in the "beg" box, "jth" number of iteration in the "thin" box and number of percentile points from "percentiles" box. Further, after typing " $*$ " in the node box, user can also ensure the convergence of the drawn samples from options like "trace," "jump," "history," "accept," "quantiles" and "auto cor". Finally we mention that in a similar way the code for the informative prior distributions can also be written and the details about the inbuilt densities in OpenBUGS can be seen from : Help $\rightarrow$ Distributions $\rightarrow$ 'Generic sampling distribution'

\section{References}

[1] E.K. Al-Hussaini, Predicting observables from a general class of distributions, J. Statist. Plann. Inference 79, 79-91, 1999.

[2] M.A.M. Ali Mousa, Inference and prediction for Pareto progressively censored data, J. Stat. Comput. Simul. 71, 163-181, 2001.

[3] M.A.M. Ali Mousa and S.A. Al-Sagheer, Bayesian prediction for progressively type-II censored data from the Rayleigh model, Comm. Statist. Theory Methods 34, 23532361, 2005.

[4] M.A.M. Ali Mousa and Z.F. Jaheen, Bayesian prediction for progressively censored data from the Burr model, Statist. Papers 43, 587-593, 2002.

[5] A. Asgharzadeh, R. Valiollahi and D. Kundu, Prediction for future failures in Weibull distribution under hybrid censoring, J. Stat. Comput. Simul. 85, 824-838, 2015.

[6] N. Balakrishnan and E. Cramer, The Art of Progressive Censoring: Applications to Reliability and Quality, Birkhäuser, 2014.

[7] N. Balakrishnan, N. Kannan, C.T. Lin and H.K.T. Ng, Point and interval estimation for Gaussian distribution, based on progressively type-II censored samples, IEEE Trans. Reliab. 52, 90-95, 2003.

[8] N. Balakrishnan and J. Mi, Existence and uniqueness of the MLEs for normal distribution based on general progressively type-II censored samples, Statist. Probab. Lett. 64, 407-414, 2003.

[9] A. Banerjee and D. Kundu, Inference based on type-II hybrid censored data from a Weibull distribution, IEEE Trans. Reliab. 57, 369-378, 2008.

[10] A. Basavalingappa, J.M. Passage, M.Y. Shen and J.R. Lloyd, Lognormal versus Weibull distribution, In IEEE International Integrated Reliability Workshop (IIRW), 2017.

[11] M.-H. Chen and Q.-M. Shao, Monte Carlo estimation of Bayesian credible and HPD intervals, J. Comput. Graph. Statist. 8, 69-92, 1999. 
[12] E.L. Crow and L. Shimizu, Lognormal Distributions: Theory and Applications, Dekker, 1988.

[13] S. Dey, S. Singh, Y.M. Tripathi and A. Asgharzadeh, Estimation and prediction for a progressively censored generalized inverted exponential distribution, Stat. Methodol. 32, 185-202, 2016.

[14] S.-R. Huang and S.-J. Wu, Bayesian estimation and prediction for Weibull model with progressive censoring, J. Stat. Comput. Simul. 82, 1607-1620, 2012.

[15] R.J. Hyndman, Computing and graphing highest density regions, Amer. Statist. 50, 120-126, 1996.

[16] Z.F. Jaheen, Prediction of progressive censored data from the Gompertz model, Comm. Statist. Simulation Comput. 32, 663-676, 2003.

[17] X. Jia, S. Nadarajah and B. Guo, The effect of mis-specification on mean and selection between the Weibull and lognormal models, Phys. A 492, 1875-1891, 2018.

[18] N.L. Johnson, S. Kotz and N. Balakrishnan, Continuous Univariate Distributions, second edition, Wiley, 1994.

[19] D. Kelly and C. Smith, Bayesian Inference for Probabilistic Risk Assessment: A Practitioner's Guidebook, Springer, 2011.

[20] H.M. Khan, M.S. Haq and S.B. Provost, Bayesian prediction for the log-normal model under type II censoring, J. Stat. Theory Appl. 5, 141-160, 2006.

[21] D. Kundu, Bayesian inference and life testing plan for Weibull distribution in presence of progressive censoring, Technometrics 50, 144-154, 2008.

$[22]$ D. Kundu and H. Howlader, Bayesian inference and prediction of the inverse Weibull distribution for type-II censored data, Comput. Statist. Data Anal. 54, 1547-1558, 2010.

[23] D. Kundu and M.Z. Raqab, Bayesian inference and prediction of order statistics for a type-II censored Weibull distribution, J. Statist. Plann. Inference 142, 41-47, 2012.

[24] J. Lawless, Statistical Models and Methods for Lifetime Data, second edition, Wiley, 2003.

[25] R.B. Leipnik, Lognormal random variables, J. Amer. Math. Soc., Series B 32, 327-347, 1991.

[26] D.V. Lindley, Approximate Bayesian methods, Trabajos de Estadistica 31, 223-245, 1980 .

[27] W.Q. Meeker and L.A. Escobar, Statistical Methods and Reliability Data, Wiley, 1998.

[28] M.M. Mohie El-Din and A.R. Shafay, One- and two-sample Bayesian prediction intervals based on progressively type-II censored data, Statist. Papers 54, 287-307, 2013.

[29] H.K.T. Ng, P.S. Chan and N. Balakrishnan, Estimation of parameters from progressively censored data using EM algorithm, Comput. Statist. Data Anal. 39, 371-386, 2002.

[30] H.K.T. Ng, P.S. Chan and N. Balakrishnan, Optimal progressive censoring plans for the Weibull distribution, Technometrics 46, 470-481, 2004.

[31] H. Panahi and A. Sayyareh, Parameter estimation and prediction of order statistics for the Burr Type XII distribution with type II censoring, J. Appl. Stat. 41, 215-232, 2014.

[32] B. Pradhan and D. Kundu, On progressively censored generalized exponential distribution, TEST 18, 497-515, 2009.

[33] M.Z. Raqab, S.A. Al-Awadhi and D. Kundu, Discriminating among Weibull, lognormal, and log-logistic distributions, Comm. Statist. Simulation Comput. 47, 13971419, 2018.

[34] S. Singh and Y.M. Tripathi, Bayesian estimation and prediction for a hybrid censored lognormal distribution, IEEE Trans. Reliab. 65, 782-795, 2016.

[35] S. Singh and Y.M. Tripathi, Estimating the parameters of an inverse Weibull distribution under progressive type-I interval censoring, Statist. Papers 59, 21-56, 2018. 
[36] S. Singh, Y.M. Tripathi and S.-J. Wu, On estimating parameters of a progressively censored lognormal distribution, J. Stat. Comput. Simul. 85, 1071-1089, 2015.

[37] S.K. Sinha, Bayesian Estimation, New Age International Publishers, 1998.

[38] A.A. Soliman, Estimation of parameters of life from progressively censored data using Burr-XII model, IEEE Trans. Reliab. 54, 34-42, 2005.

[39] L. Tierney and J.B. Kadane, Accurate approximations for posterior moments and marginal densities, J. Amer. Statist. Assoc. 81, 82-86, 1986.

[40] N. Turkkan and T. Pham-Gia, Computation of the highest posterior density interval in Bayesian analysis, J. Stat. Comput. Simul. 44, 243-250, 1993. 